\title{
Observation of an "ABC" Effect in Proton-Proton Collisions
}

S. Dymov, ${ }^{1,2, *}$ M. Hartmann, ${ }^{3,4}$ A. Kacharava, ${ }^{3,4}$ A. Khoukaz, ${ }^{5}$ V. Komarov, ${ }^{1}$ P. Kulessa, ${ }^{6}$ A. Kulikov, ${ }^{1}$ V. Kurbatov, ${ }^{1}$ G. Macharashvili, ${ }^{1,7}$ S. Merzliakov, ${ }^{1,3,4}$ M. Mielke, ${ }^{5}$ S. Mikirtychiants,,${ }^{3,4,8}$ M. Nekipelov,,${ }^{3,4}$ M. Nioradze, ${ }^{7}$ H. Ohm, ${ }^{3,4}$ F. Rathmann, ${ }^{3,4}$ H. Ströher, ${ }^{3,4}$ D. Tsirkov, ${ }^{1,3,4}$ Yu. Uzikov, ${ }^{1}$ Yu. Valdau, ${ }^{3,4,8}$ C. Wilkin, ${ }^{9}$

S. Yaschenko, ${ }^{1,2}$ and B. Zalikhanov ${ }^{1}$

${ }^{1}$ Laboratory of Nuclear Problems, Joint Institute for Nuclear Research, RU-141980 Dubna, Russia

${ }^{2}$ Physikalisches Institut II, Universität Erlangen-Nürnberg, D-91058 Erlangen, Germany

${ }^{3}$ Institut für Kernphysik, Forschungszentrum Jülich GmbH, D-52425 Jülich, Germany

${ }^{4}$ Jülich Centre for Hadron Physics, D-52425 Jülich, Germany

${ }^{5}$ Institut für Kernphysik, Universität Münster, D-48149 Münster, Germany

${ }^{6} \mathrm{H}$. Niewodniczański Institute of Nuclear Physics PAN, PL-31342 Kraków, Poland

${ }^{7}$ High Energy Physics Institute, Tbilisi State University, GE-0186 Tbilisi, Georgia

${ }^{8}$ High Energy Physics Department, Petersburg Nuclear Physics Institute, RU-188350 Gatchina, Russia

${ }^{9}$ Physics and Astronomy Department, UCL, London, WCIE 6BT, United Kingdom

(Received 4 February 2009; published 13 May 2009)

\begin{abstract}
The cross section for inclusive multipion production in the $p p \rightarrow p p X$ reaction was measured at COSY-ANKE at four beam energies, $0.8,1.1,1.4$, and $2.0 \mathrm{GeV}$, for low excitation energy in the final $p p$ system, such that the diproton quasiparticle is in the ${ }^{1} S_{0}$ state. At the three higher energies, the missingmass $M_{X}$ spectra show a strong enhancement at low $M_{X}$, corresponding to an Abashian-Booth-Crowe effect that moves steadily to larger values as the energy is increased. Despite the missing-mass structure looking very different at $0.8 \mathrm{GeV}$, the variation with $M_{X}$ and beam energy are consistent with two-pion production being mediated through the excitation of two $\Delta(1232)$ isobars, coupled to $S$ and $D$ states of the initial $p p$ system. There is no sign of any resonancelike structure in the energy dependence of the type recently observed for the $p n \rightarrow d \pi^{0} \pi^{0}$ total cross section.
\end{abstract}

DOI: 10.1103/PhysRevLett.102.192301

PACS numbers: 13.75.Cs, 14.40.Aq, 25.40.Qa

It is known on both experimental and theoretical grounds that there is no scalar $\pi \pi$ resonance with a low mass [1]. However, in a wide variety of missing-mass experiments, $p d \rightarrow{ }^{3} \mathrm{He} X, d d \rightarrow{ }^{4} \mathrm{He} X$, and $n p \rightarrow d X$, carried out in the $1 \mathrm{GeV} /$ nucleon region, a sharp peak is found for an invariant mass of the undetected particles $M_{X} \approx 310-330 \mathrm{MeV} / c^{2}$ [2-6]. The copious production combined with its low mass means that the system $X$ consists of pion pairs with small excitation energy and hence dominantly in a relative $s$ wave. The observations of a peak in the $\pi \pi$ spectrum when there is no resonance has presented a puzzle since its first discovery by Abashian, Booth, and Crowe (ABC) almost 50 years ago [2]. If the $\mathrm{ABC}$ anomaly is not a resonance, it must be a kinematic reflection of the production mechanism and, to investigate it further, one should look at its production in the simplest systems, i.e., in nucleon-nucleon collisions.

Though seen clearly in the zero-degree momentum spectrum of the deuteron from $n p \rightarrow d X$ [6], the missing-mass resolution is here degraded by the momentum spread of the neutron beam. To avoid this, the quasifree $p d \rightarrow$ $d \pi^{0} \pi^{0} p_{\text {sp }}$ reaction has been measured semiexclusively at beam energies of $T_{p}=1.03$ and $1.35 \mathrm{GeV}$, with just the spectator proton $p_{\mathrm{sp}}$ escaping detection [7]. By exploiting the Fermi motion of the struck neutron, the $p n \rightarrow d \pi^{0} \pi^{0}$ cross section could be extracted at a variety of c.m. energies. In addition to observing clean ABC peaks, a remark- able energy dependence was found, with a resonancelike structure in the total cross section [7].

The conventional view is that $\mathrm{ABC}$ production in nucleon-nucleon collisions is mediated by the excitation and decay of two separate $\Delta$ isobars $[8,9]$, though it is not clear how such models could give rise to the observed sharp energy dependence of $p n \rightarrow d \pi^{0} \pi^{0}$. The dominant contribution to the amplitudes arises when the two $\Delta$ are in a relative $S$ wave. For the production of an $I_{\pi \pi}=0$ pion pair, isospin conservation requires $I_{\Delta \Delta}=0$. It follows from the generalized Pauli principle that the two isobars must then couple to a total spin of $S_{\Delta \Delta}=1$ or 3 . In contrast, for $p p \rightarrow \Delta \Delta \rightarrow p p \pi \pi, I_{\Delta \Delta}=1$ so that $S_{\Delta \Delta}=$ 0 or 2 , and an $s$-wave pion pair can have $I_{\pi \pi}=0$ or 2 . The investigation of the $\mathrm{ABC}$ phenomenon in $n p$ and $p p$ collisions is therefore largely complementary, and extra information might be gleaned by looking at $p p \rightarrow p p X$ in greater detail. Though there are data on this reaction at $T_{p}=1.5$ and $1.8 \mathrm{GeV}$, the authors concentrated on possible substructure [10].

The aim of the present experiment is the measurement of the $p p \rightarrow\{p p\}_{S} X$ reaction when the excitation energy in the final diproton system is low, $E_{p p}<3 \mathrm{MeV}$. This quasiparticle, denoted by $\{p p\}_{s}$, is almost exclusively in the ${ }^{1} S_{0}$ state. Under these conditions, the kinematics are similar to those of $p n \rightarrow d X$, which facilitates a comparison of the two reactions. Of the four proton beam energies used, 
$T_{p}=0.8,1.1,1.4$, and $2.0 \mathrm{GeV}$, the lowest is well below the nominal $\Delta \Delta$ threshold, and by $2 \mathrm{GeV}$, much of the $\Delta$ strength has passed.

The measurement of single and multipion production in $p p$ collisions was performed using the ANKE magnetic spectrometer [11], positioned at an internal target station of the Cooler Synchrotron (COSY) storage ring of the Forschungszentrum Jülich. The single pion results have already been reported [12]. The proton beam was directed at a hydrogen cluster-jet target with an areal density of $2 \times$ $10^{14}$ atoms $/ \mathrm{cm}^{2}$. The resulting positively charged particles were recorded in the ANKE forward detector system, consisting of three multiwire proportional chambers (MWPCs) and a two-layer scintillation hodoscope, the whole giving a horizontal acceptance of $12^{\circ}$ and $\pm 3.5^{\circ}$ vertically. The particle momenta, deduced by tracing through the analyzing magnetic field, had typical uncertainties of $\approx 1 \%$. The emerging proton pairs were identified by evaluating the difference $\Delta t$ between the times of flight measured with the hodoscope and those calculated from the particle momenta, assuming the particles to be protons [12].

Figure 1 shows the spectrum in the square of the missing mass of the $p p \rightarrow p p X$ reaction at $1.4 \mathrm{GeV}$. Selecting $p p$ events on the basis of the $\Delta t$ cut eliminates almost all $d \pi^{+}$ pairs, leaving a background of less than $0.1 \%$. To ensure that the two protons are dominantly in the ${ }^{1} S_{0}$ state, a further cut on the $p p$ excitation energy, $E_{p p}<3 \mathrm{MeV}$, was imposed. After weighting by detection efficiency, such pairs are distributed isotropically in their rest frame, as expected for an $S$ wave. The $E_{p p}$ distributions also follow closely the expected $S$-wave final-state-interaction

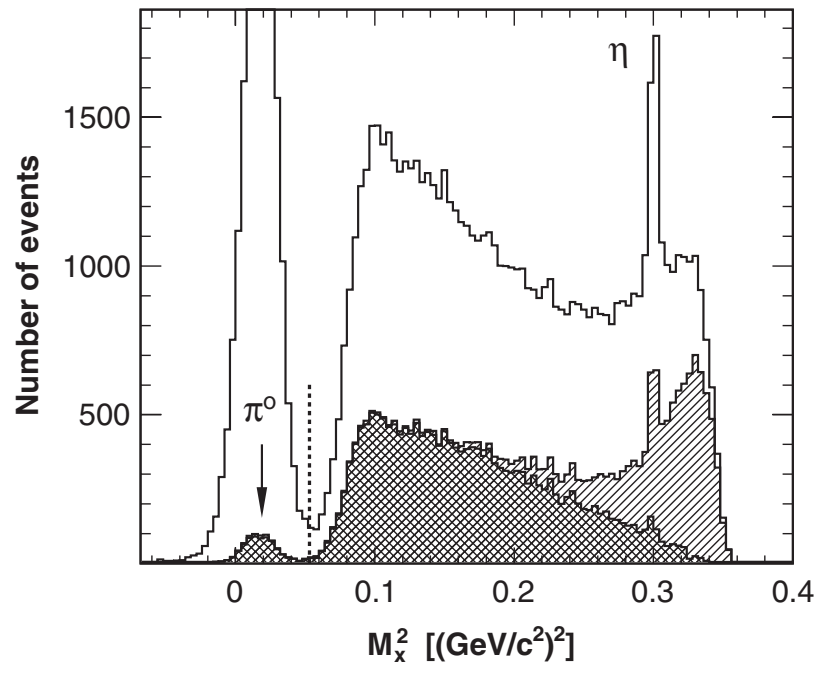

FIG. 1. Distribution in missing mass squared of the $p p \rightarrow$ $p p X$ reaction at $1.4 \mathrm{GeV}$. Imposing a $\Delta t$ cut to select the two protons gives the open histogram. The lightly shaded plot corresponds to the additional requirement that $E_{p p}<3 \mathrm{MeV}$, and the heavy shading reflects the further $\cos \vartheta_{p p}>0.95$ cut to match the overall ANKE acceptance. The positions of the $\pi^{0}$ and $\eta$ peak are indicated, as is the two-pion threshold (dotted line). behavior. Since for low missing masses the c.m. angle of the diproton, $\vartheta_{p p}$, is strongly limited by the ANKE acceptance, we imposed the extra cut $\cos \vartheta_{p p}>0.95$ on the whole spectrum. Apart from peaks corresponding to the production of single $\pi^{0}$ and $\eta$ mesons, the distribution shows a rapid rise from the two-pion threshold with a gentle decrease towards the upper kinematic limit.

The development of the counting rate distribution with energy is displayed in Fig. 2 for the multipion region. Also shown there are the results of a Monte Carlo simulation of the detector and analysis code, using four-body $p p \pi \pi$ phase space with inclusion of $p p$ final state interaction [12]. The effects of momentum resolution are included and the same cuts applied as in the experiment. In no case does this approach reproduce the data.

A simulation was used to deduce the differential cross sections of Fig. 3 from the counting rate data, taking as input the double- $\Delta$ model to be discussed with Eq. (3). The simulation also defines the momentum reconstruction uncertainties, and this leads to a $\pi^{0}$ peak whose width is compatible with that shown in Fig. 1. The requisite luminosity was derived from the simultaneous measurement of $p p$ elastic scattering [12].

As shown in Fig. 3, the strength at $0.8 \mathrm{GeV}$ is pushed towards the maximum missing mass, while at the higher three energies, there is a peak of the $\mathrm{ABC}$ type at low $M_{X}$ and no sign of any enhancement at large masses. Above its threshold, there are also contributions from $\eta$ production, with cross sections integrated over our cuts of $\sigma_{\eta}=4.3 \pm$ $0.8 \mathrm{nb}$ at $1.4 \mathrm{GeV}$ and $4.5 \pm 0.9 \mathrm{nb}$ at $2.0 \mathrm{GeV}$. The $\mathrm{ABC}$ position moves steadily to the right and gets broader as $T_{p}$

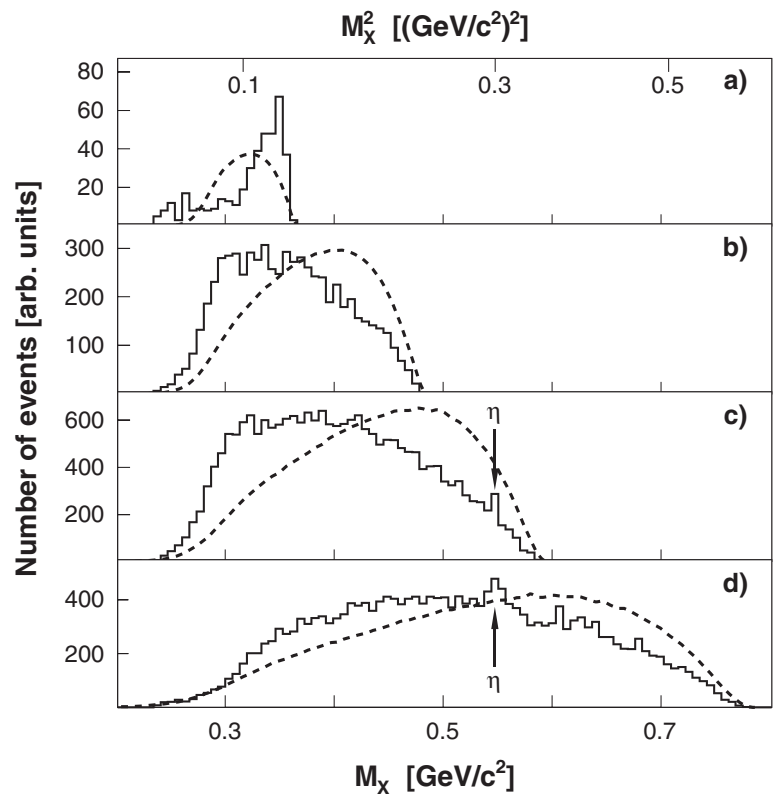

FIG. 2. Distribution in missing mass of the $p p \rightarrow\{p p\}_{s} X$ reaction for $E_{p p}<3 \mathrm{MeV}$ and $\cos \vartheta_{p p}>0.95$ at (a) 0.8 , (b) 1.1 , (c) 1.4 , and (d) $2.0 \mathrm{GeV}$. The $\eta$ signal is seen at the expected position for the two higher energies. The curves represent normalized simulations within a phase-space model. 


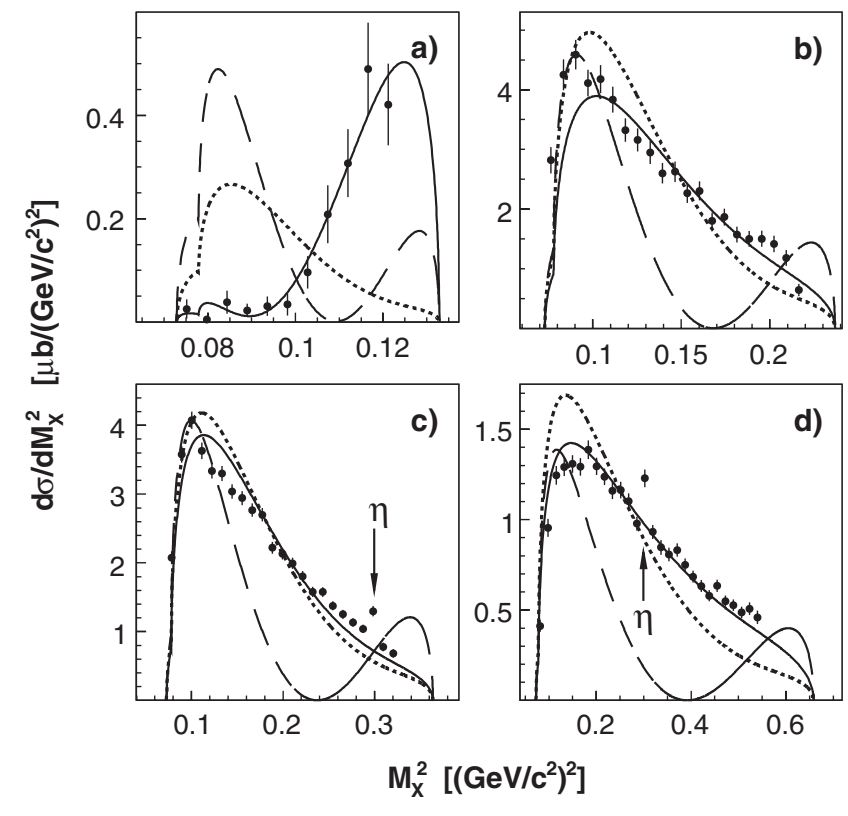

FIG. 3. The $p p \rightarrow p p X$ differential cross section with statistical errors as a function of the square of the missing mass at (a) 0.8 , (b) 1.1 , (c) 1.4 , and (d) $2.0 \mathrm{GeV}$ for $E_{p p}<3 \mathrm{MeV}$ and $\cos \vartheta_{p p}>0.95$. The $\eta$ peaks are indicated. Events were simulated using Eq. (3) with $A_{D}=0$ (long dashes), with $A_{S}=0$ (short-dashed), and with the best fit of Table I (solid line).

increases above $1.1 \mathrm{GeV}$, which means that the phenomenon does depend upon the kinematics, which is not the case of a resonance of fixed mass and width. The peak seems to be rather clearer than that observed at Saclay at 1.5 and $1.8 \mathrm{GeV}$ [10]. Although a previous exclusive measurement of $p p \rightarrow p p \pi^{+} \pi^{-}$at $0.8 \mathrm{GeV}$ [13] did not study in detail our kinematic configuration, the two data sets are consistent in their region of overlap.

The total cross section for the production of three pions in $p p$ collisions at $1.35 \mathrm{GeV}$ is about $0.6 \%$ of that for two pions [14], and so it is only at $2 \mathrm{GeV}$ that there might be any significant contribution to the data from the $p p \rightarrow$ $p p \pi \pi \pi$ reaction. The modeling of the results must therefore be based upon the assumption that two-pion production dominates.

A $\Delta \Delta$ model applied to two-meson production in the $p n \rightarrow d X$ or $d d \rightarrow{ }^{4} \mathrm{He} X$ reaction allows the momentum transfer to be shared between the nucleons, facilitating the formation of the observed deuteron or ${ }^{4} \mathrm{He}$. The strong peaking of the $\Delta$ decay in the forward and backward directions also enhances this sticking factor. It is therefore particularly suited to our conditions but might not be appropriate for higher $E_{p p}$ or larger production angles. A full estimation of $p p \rightarrow\{p p\}_{s} \pi \pi$ in such a model depends sensitively upon the exchange mesons and form factors, etc. Nevertheless, the general structure can be written down if we assume that the intermediate $\Delta$ are in a relative $S$ wave so that the initial protons are in the ${ }^{1} S_{0}$ or ${ }^{1} D_{2}$ wave. Since the $p$-wave nature of each $\Delta$ decay necessarily involves a momentum factor, the obvious ansatz for the matrix element is

$$
\mathcal{M}=A_{S}\left(\vec{k}_{1}^{\prime} \cdot \vec{k}_{2}^{\prime}\right)+\frac{1}{2} A_{D}\left[3\left(\hat{p} \cdot \vec{k}_{1}^{\prime}\right)\left(\hat{p} \cdot \vec{k}_{2}^{\prime}\right)-\vec{k}_{1}^{\prime} \cdot \vec{k}_{2}^{\prime}\right]
$$

where $\vec{p}$ is the c.m. proton beam momentum and $\vec{k}_{i}^{\prime}$ is the relative momentum of the $i$ th pion with respect to one of the recoiling protons. The $S$ - and $D$-wave amplitudes $A_{S}$ and $A_{D}$ are scalar functions that may depend strongly upon the kinematic variables because of the excitation of the two $\Delta$, though we shall neglect any variation with $M_{X}$. In terms of the relative $\pi \pi$ momentum, $2 \vec{q}$, and that of the diproton, $-\vec{k}$, the pion c.m. momenta are $\vec{k}_{1,2}=\frac{1}{2} \vec{k} \pm \vec{q}$, and the matrix element becomes

$$
\begin{aligned}
\mathcal{M}= & A_{S}\left(\alpha^{2} k^{2}-\beta^{2} q^{2}\right)+\frac{1}{2} A_{D}\left[3\left(\alpha^{2} k_{z}^{2}-\beta^{2} q_{z}^{2}\right)\right. \\
& \left.-\left(\alpha^{2} k^{2}-\beta^{2} q^{2}\right)\right],
\end{aligned}
$$

where the $z$-direction is taken along $\vec{p}$. In this approximation, $q^{2}$ and $k^{2}$ are linked by energy conservation. For simplicity of presentation, a Galilean transformation is used to evaluate the recoil factors $\alpha=\frac{1}{2}(m+2 \mu) /(m+$ $\mu)$ and $\beta=m /(m+\mu)$ in terms of the pion $(\mu)$ and proton $(m)$ masses. Only the part of the matrix element proportional to $3 q_{z}^{2}-q^{2}$ corresponds to a $d$ wave in the $\pi \pi$ system, and this contributes very little to the $\mathrm{ABC}$ peak, which is $s$ wave in nature.

Since the direction of the $\pi \pi$ relative momentum is not observed in a missing-mass experiment, $|\mathcal{M}|^{2}$ must be averaged over the angles of $\vec{q}$;

$$
\begin{aligned}
\left\langle|\mathcal{M}|^{2}\right\rangle= & \left|A_{S}\left(\alpha^{2} k^{2}-\beta^{2} q^{2}\right)+\frac{1}{2} A_{D} \alpha^{2}\left(3 k_{z}^{2}-k^{2}\right)\right|^{2} \\
& +\frac{1}{5}\left|A_{D}\right|^{2} \beta^{4} q^{4} .
\end{aligned}
$$

Figure 3 shows the results of implementing the simple double- $\Delta$ model of Eq. (3). Since the charges of the pions were not measured, we assumed an $I_{\pi \pi}=0$ ratio of $\pi^{+} \pi^{-}: \pi^{0} \pi^{0}=2: 1$, with the corresponding masses being used in the two phase spaces. If there were only $S$-wave production, $\left\langle|\mathcal{M}|^{2}\right\rangle$ would be maximal at $q^{2}=0$ (the ABC bump) and at $k^{2}=0$ (the maximum allowed missing mass), with a deep valley between these two structures. Such behavior is seen for $d d \rightarrow{ }^{4} \mathrm{He} X[4,5]$. The $A_{D}=0$ case is illustrated in Fig. 3, as is that of $A_{S}=0$, which gives a broad maximum at low $\pi \pi$ invariant masses and no sign of any high mass bump.

The general shapes of the spectra at the three higher energies are qualitatively reproduced by the pure $D$-wave model. However, the peaking towards maximum $M_{X}$ in the $0.8 \mathrm{GeV}$ data shows the necessity for a substantial $S$-wave contribution at this energy. To investigate this, the uncorrected data of Fig. 2 were fitted to the Monte Carlo simulations using as input Eq. (3) with free values of $A_{S}$ and $A_{D}$. The parameters that best reproduce the spectra are given in Table I along with the resulting integrated cross sections. 
TABLE I. Cross section $\sigma_{X}$ for the $p p \rightarrow\{p p\}_{s} X$ reaction integrated over $\cos \vartheta_{p p}>0.95, E_{p p}<3 \mathrm{MeV}$ and over $M_{X}$ up to the kinematical limit. The experimental acceptance is limited to less than $M_{X}^{\max }$, and $\sigma_{X}$ is obtained by correcting the measured cross section by an amount $\xi$, as suggested by fits of the $\Delta \Delta$ model to the differential data. In addition to the given statistical errors, there are systematic uncertainties of $7 \%$, coming principally from the luminosity and acceptance evaluations [12]. The parameters $A_{S}$ and $A_{D}$ are determined by fitting the spectra away from regions of rapid variation, where resolution questions arise, and at too high masses to avoid the tail from $\rho$ production. The results for $A_{D}$ are normalized at $1.1 \mathrm{GeV}$.

\begin{tabular}{cccccc}
\hline \hline $\begin{array}{c}T_{p} \\
\mathrm{GeV}\end{array}$ & $\begin{array}{c}\left(M_{X}^{\max }\right)^{2} \\
\left(\mathrm{GeV} / c^{2}\right)^{2}\end{array}$ & \multicolumn{1}{c}{$\begin{array}{c}\sigma_{X} \\
\mathrm{nb}\end{array}$} & $\begin{array}{c}\xi \\
\%\end{array}$ & \multicolumn{1}{c}{$A_{S} / A_{D}$} & $A_{D}$ \\
\hline 0.8 & 0.123 & $12 \pm 2$ & 34 & $-1.23 \pm 0.10$ & $1.09 \pm 0.05$ \\
1.1 & 0.219 & $389 \pm 6$ & 2.3 & $-0.20 \pm 0.03$ & 1.00 \\
1.4 & 0.326 & $563 \pm 6$ & 2.4 & $-0.10 \pm 0.03$ & $0.48 \pm 0.01$ \\
2.0 & 0.549 & $456 \pm 5$ & 5.8 & $-0.23 \pm 0.02$ & $0.17 \pm 0.01$ \\
\hline \hline
\end{tabular}

The values of $A_{D}$ fall steadily with energy, but only at $0.8 \mathrm{GeV}$ is $A_{S} / A_{D}$ required to be large. The description of the data shown in Fig. 3 is much improved, especially at $0.8 \mathrm{GeV}$, but the model would have to be refined so as to reproduce the details of the $\mathrm{ABC}$ peaks seen at 1.1 and $1.4 \mathrm{GeV}$.

Within the $S$-wave $\Delta \Delta$ approach, the matrix element of Eq. (1) allows for both $A_{S}$ and $A_{D}$ terms. If two-pion production were driven instead by the excitation of $S$-wave $N^{*}(1440) N$ pairs, where $N^{*}$ is the Roper resonance, an $A_{S}$ term is generated through the double $p$-wave cascade decay $N^{*}(1440) \rightarrow \Delta \pi \rightarrow p \pi \pi$. However, in this model, it follows that $A_{D}=0$. Contributions from both $N^{*} N$ and $\Delta \Delta$ might provide an explanation for the energy dependence of $A_{S} / A_{D}$ shown in Table I. It should be noted that the $\Delta \Delta$ model would lead to an ABC effect also in $p p \rightarrow$ $\{n n\}_{S} \pi^{+} \pi^{+}$, which is forbidden in the $N^{*} N$ model. If the $\mathrm{ABC}$ is not a resonance, it does not have to have a definite isospin, and previous studies of the effect were not sensitive to any $I_{\pi \pi}=2$ component.

The special quasiparticle $p p \rightarrow\{p p\}_{S} X$ kinematics are very similar to those of $p n \rightarrow d \pi^{0} \pi^{0}$ and so a comparison with the CELSIUS-WASA data [7] is instructive. In neither case is there an enhancement at high masses and the ABC peak itself seems to be narrower in $p n$ collisions. The measurement of the Fermi momentum when using a deuterium target allowed the determination of cross sections as a function of the laboratory kinetic energy $T_{p}$. The results suggest a resonancelike structure with a mass of $2.36 \mathrm{GeV} / c^{2}$ and width of $\Gamma \approx 80 \mathrm{MeV} / c^{2} \quad\left(T_{p} \approx\right.$ $1.09 \mathrm{GeV}, \Gamma_{T}=0.20 \mathrm{GeV}$ ) [7]. Although our energy intervals are rather wide, no such behavior is obvious in the values given in Table I. Since there are different spinisospin selection rules in the two cases, this would suggest a strong spin or isospin dependence of the production mechanism.
In summary, we have measured the differential cross section for the $p p \rightarrow\{p p\}_{S} X$ reaction at four beam energies from 0.8 to $2.0 \mathrm{GeV}$ under specific kinematic conditions. The proton-proton excitation energy is below $3 \mathrm{MeV}$, and hence the diproton is dominantly in the ${ }^{1} S_{0}$ state, and the c.m. angle between the diproton momentum and the beam axis is less than $18^{\circ}$. Strong deviations from phase space are observed in the missing-mass variable, with a peak in $M_{X}$ whose position varies with beam energy, and no broad bump at maximum missing mass of the type observed in $d d \rightarrow{ }^{4} \mathrm{HeX}[4,5]$.

The variation of our data with energy point to the $A B C$ effect in $p p$ collisions at small angles being the result of the production mechanism and this is backed up by a phenomenological description. In view of the simple spin structure, after assuming that each of the two pions is emitted in a $p$ wave, there are only two amplitudes to be considered. Except for the $0.8 \mathrm{GeV}$ data, the bulk of the $M_{X}$ spectra are consistent with a dominant $D$-wave coupling to the incident protons, whereas at the lowest energy, the $S$ wave is significant. A fully microscopic model would be required to build upon the successes of this approach.

In contrast to the CELSIUS-WASA work on $p n \rightarrow$ $d \pi^{0} \pi^{0}$, we certainly have no evidence for an $I=1 \mathrm{di}$ baryon, and this is consistent with robust $p p$ phase shift analyses in this region [15]. On the other hand, the $n p$ database is much poorer above $1 \mathrm{GeV}$ and an $I=0$ dibaryon could not be rigorously excluded on these grounds.

We are grateful to other members of the ANKE Collaboration for their help with this experiment and to the COSY crew for providing such good working conditions. This work was supported in part by the BMBF grant ANKE COSY-JINR, HGF-VIQCD, and JCHP FFE.

*s.dymov@fz-juelich.de

[1] S. Spanier, N. A. Törnqvist, and W. M. Yao et al. (PDG), J. Phys. G 33, 1 (2006).

[2] A. Abashian, N.E. Booth, and K. M. Crowe, Phys. Rev. Lett. 5, 258 (1960).

[3] J. Banaigs et al., Nucl. Phys. B67, 1 (1973).

[4] J. Banaigs et al., Nucl. Phys. B105, 52 (1976).

[5] R. Wurzinger et al., Phys. Lett. B 445, 423 (1999).

[6] F. Plouin et al., Nucl. Phys. A 302, 413 (1978).

[7] M. Bashkanov et al., Phys. Rev. Lett. 102, 052301 (2009).

[8] T. Risser and M. D. Shuster, Phys. Lett. B 43, 68 (1973).

[9] C. A. Mosbacher and F. Osterfeld, in Baryons '98, edited by D.W. Menze and B. Metsch (World Scientific, Singapore, 1999), p. 609.

[10] J. Yonnet et al., Phys. Rev. C 63, 014001 (2000).

[11] S. Barsov et al., Nucl. Instrum. Methods Phys. Res., Sect. A 462, 364 (2001).

[12] S. Dymov et al., Phys. Lett. B 635, 270 (2006); V. Kurbatov et al., Phys. Lett. B 661, 22 (2008).

[13] S. Abd El-Bary et al., Eur. Phys. J. A 37, 267 (2008).

[14] C. Pauly et al., Phys. Lett. B 649, 122 (2007).

[15] R. A. Arndt, I. I. Strakovsky, and R. L. Workman, Phys. Rev. C 62, 034005 (2000); http://gwdac.phys.gwu.edu/. 